\title{
Some aspects of isospin breaking in kaon decays
}

\author{
Helmut Neufeld ${ }^{* \dagger}$ \\ Faculty of Physics, University of Vienna, Boltzmanngasse 5, A-1090 Wien, Austria \\ E-mail: Helmut.Neufeld@univie.ac.at
}

\begin{abstract}
I discuss a recent calculation of the radiative corrections for all $K_{\ell 3}$ decay modes. I review a new update of the determination of the isospin breaking parameter $\delta_{\mathrm{SU}(2)}^{K \pi}$ using current information on the electromagnetic contributions to the pseudoscalar masses. I compare the theoretical result with an "experimental" determination of this parameter from $K_{\ell 3}$ data. Finally, I discuss recent theoretical results on isospin breaking effects in the $K_{\ell 3}$ scalar form factors.
\end{abstract}

6th International Workshop on Chiral Dynamics

July 6-10 2009

Bern, Switzerland

\footnotetext{
${ }^{*}$ Postersession.

†Supported in part by EU Contract MRTN-CT-2006-035482, "FLAVIAnet". I wish to thank Vincenzo Cirigliano, Maurizio Giannotti and Andreas Kastner for a fruitful collaboration.
} 


\section{1. $K_{\ell 3}$ decays}

In the standard model, transitions of quark flavours in weak interactions are described by a unitary matrix, the famous Cabibbo-Kobayshi-Maskawa (CKM) mixing matrix. The $K_{\ell 3}$ decays

$$
K^{0}\left(p_{K}\right) \rightarrow \pi^{-}\left(p_{\pi}\right) \ell^{+}\left(p_{\ell}\right) v_{\ell}\left(p_{v}\right), \quad K^{+}\left(p_{K}\right) \rightarrow \pi^{0}\left(p_{\pi}\right) \ell^{+}\left(p_{\ell}\right) v_{\ell}\left(p_{v}\right)
$$

(and their charge conjugate modes) are an important source of information on the the CKM matrix element $V_{u s}$ which is needed for a test of the unitarity relation $\left|V_{u d}\right|^{2}+\left|V_{u s}\right|^{2}+\left|V_{u b}\right|^{2}=1$. These decays also provide competitive probes of lepton universality, the ratios of light quark masses and the dependence of the associated form factors on the momentum transfer to the lepton pair. With the present high-statistics experiments (see [1] and references therein), the Cabibbo angle $V_{u s}$ can be measured with a precision at the subpercent level. In order to fully exploit these amazing experimental achievements, it is mandatory to have full theoretical control of these decays at the percent level or better. This requires in particular a proper treatment of electromagnetic corrections and other isospin breaking effects at all stages of the analysis.

Including electromagnetic corrections, the fully inclusive decay rate for all four $K_{\ell 3}$ modes $\left(K=K^{ \pm}, K^{0}, \overline{K^{0}} ; \ell=e, \mu\right)$ can be written as

$$
\Gamma\left(K_{\ell 3[\gamma]}\right)=\frac{G_{\mathrm{F}}^{2}\left|V_{u s}\right|^{2} M_{K}^{5} C_{K}^{2}}{128 \pi^{3}} S_{\mathrm{EW}}\left|f_{+}^{K^{0} \pi^{-}}(0)\right|^{2} I_{K \ell}^{(0)}\left(\lambda_{i}\right)\left(1+\delta_{\mathrm{EM}}^{K \ell}+\delta_{\mathrm{SU}(2)}^{K \pi}\right) .
$$

In this formula, $G_{F}$ is the Fermi decay constant as extracted from muon decays, the Clebsch-Gordan coefficient $C_{K}$ differs for neutral and charged kaons $\left(C_{K}=1\right.$ for $K_{\ell 3}^{0}$ and $C_{K}=1 / \sqrt{2}$ for $\left.K_{\ell 3}^{+}\right)$, while $I_{K \ell}^{(0)}$ is a phase-space integral depending on slope and curvature of the form factors $f_{ \pm}^{K \pi}(t)$. As usual, the vector form factor of the $K^{0}$ decay at zero momentum transfer has been pulled out. The strong isospin breaking correction is defined as $\delta_{\mathrm{SU}(2)}^{K \pi}=\left(f_{+}^{K \pi}(0) / f_{+}^{K^{0} \pi^{-}}(0)\right)^{2}-1$. The electroweak short-distance enhancement factor [2,3]

$$
S_{\mathrm{EW}}=1+\frac{2 \alpha}{\pi}\left(1-\frac{\alpha_{s}}{4 \pi}\right) \log \frac{M_{Z}}{M_{\rho}}+\mathscr{O}\left(\frac{\alpha \alpha_{s}}{\pi^{2}}\right)=1.0223 \pm 0.0005
$$

is universal for all semileptonic decays. The long-distance electromagnetic corrections $\delta_{\mathrm{EM}}^{K \ell}=$ $\delta_{\mathrm{EM}}^{K \ell}\left(\mathscr{D}_{3}\right)+\delta_{\mathrm{EM}}^{K \ell}\left(\mathscr{D}_{4-3}\right)$ receive contributions from three-particle and four-particle final states.

\section{Long-distance electromagnetic corrections}

The appropriate theoretical tool to analyze electromagnetic corrections is provided by chiral perturbation theory with virtual photons $[4,5,6]$ and leptons [7]. Using this theoretical framework, the calculation of electromagnetic contributions to $\mathscr{O}\left(e^{2} p^{2}\right)$ in $K_{\ell 3}$ decays was presented in [8]. Based on this analysis, full numerical results on the $K_{e 3}$ decay modes were given in [9] adopting a specific prescription for treating real photon emission and a specific factorization scheme for soft photons. This approach resulted in the partial inclusion of higher order terms in the chiral expansion.

In a recent publication [10] we have extended our numerical analysis of electromagnetic corrections also to a complete study of $K_{\mu 3}$ decays. At the same time, we have updated our previous 
results for the $K_{e 3}$ modes using the new estimates $[11,12]$ of the electromagnetic low-energy constants which affect the structure-dependent electromagnetic contributions. Rather than using the soft-photon factorization procedure of [8], we were working to fixed chiral order $e^{2} p^{2}$. In contrast to our previous studies, we were also employing a fully inclusive prescription for real photon emission being more appropriate for comparison with experimental results.

Table 1 summarizes our numerical results for the long-distance radiative corrections. Two characteristic features can be understood by qualitative arguments. Firstly, the electromagnetic corrections for the neutral $K$ decays are expected to be positive and sizable on account of the final state Coulomb interaction between $\ell^{+}$and $\pi^{-}$producing a correction factor of $\pi \alpha / v_{\ell^{+}}^{\text {rel }} \pi^{-} \sim 2 \%$ over most of the Dalitz plot. While the exact correction and the relative size of $K_{\mu 3}^{0}$ and $K_{e 3}^{0}$ depend on other effects such as the emission of real photons, the qualitative expectation based on the Coulomb interaction is confirmed by the detailed calculation. Secondly, the large hierarchy $\delta_{\mathrm{EM}}^{K \mu}\left(\mathscr{D}_{4-3}\right) \ll \delta_{\mathrm{EM}}^{K e}\left(\mathscr{D}_{4-3}\right)$ admits a simple interpretation in terms of bremsstrahlung off the charged lepton in the final state. The probability of emitting soft photons is a function of the lepton velocity $v_{\ell}$ which becomes logarithmically singular as $v_{\ell} \rightarrow 1$, thus enhancing the electron emission. For typical values of $v_{\ell}$ in $\mathscr{D}_{4-3}$, the semiclassical emission probability implies $\delta_{\mathrm{EM}}^{K e}\left(\mathscr{D}_{4-3}\right) / \delta_{\mathrm{EM}}^{K \mu}\left(\mathscr{D}_{4-3}\right) \sim 20 \rightarrow 40$.

\begin{tabular}{|l|c|c|c|}
\hline & $\delta_{\mathrm{EM}}^{K \ell}\left(\mathscr{D}_{3}\right)(\%)$ & $\delta_{\mathrm{EM}}^{K \ell}\left(\mathscr{D}_{4-3}\right)(\%)$ & $\delta_{\mathrm{EM}}^{K \ell}(\%)$ \\
\hline$K_{e 3}^{0}$ & 0.50 & 0.49 & $0.99 \pm 0.22$ \\
$K_{e 3}^{ \pm}$ & -0.35 & 0.45 & $0.10 \pm 0.25$ \\
$K_{\mu 3}^{0}$ & 1.38 & 0.02 & $1.40 \pm 0.22$ \\
$K_{\mu 3}^{ \pm}$ & 0.007 & 0.009 & $0.016 \pm 0.25$ \\
\hline
\end{tabular}

Table 1: Electromagnetic corrections to the $K_{\ell 3}$ decay rates given in [10], calculated to fixed order in chiral perturbation theory. $\mathscr{D}_{3}$ refers to three-particle phase space, $\mathscr{D}_{4}$ to four-body kinematics.

The theoretical uncertainties assigned to the $\delta_{\mathrm{EM}}^{K \ell}$ in Table 1 arise from two sources: the input parameters (low-energy constants and form factor parameters) used in the calculation and unknown higher order terms in the chiral expansion (the latter would require a complete analysis at order $e^{2} p^{4}$ ). The experimental errors of $\lambda_{+}, \lambda_{+}^{\prime \prime}$ and $\lambda_{0}$ induce a fractional uncertainty in $\delta_{\mathrm{EM}}^{K \ell}$ well below the percent level. This source of uncertainty can safely be neglected. The structure dependent contributions to the electromagnetic corrections are determined by the electromagnetic low-energy couplings $X_{1}$ and $X_{6}^{\text {phys }}$. Taking the central values from [12] and assigning very conservative $100 \%$ fractional uncertainties to these parameters, we find an absolute uncertainty of $\pm 0.11 \%$ in $\delta_{\mathrm{EM}}^{K^{0} \ell}$ and of $\pm 0.16 \%$ in $\delta_{\mathrm{EM}}^{K^{ \pm} \ell}$.

For an estimate of the uncertainties due to higher order chiral corrections not included in the analysis of [10], it is useful to decompose the EM corrections $\delta^{K \ell}$ in terms of parameters $\delta_{1,2,3,4}$,

$$
\begin{array}{ll}
\delta^{K^{0} e}=\delta_{1}+\delta_{2}+\delta_{3}+\delta_{4}, & \delta^{K^{0} \mu}=\delta_{1}+\delta_{2}-\delta_{3}-\delta_{4}, \\
\delta^{K^{ \pm} e}=\delta_{1}-\delta_{2}+\delta_{3}-\delta_{4}, & \delta^{K^{ \pm} \mu}=\delta_{1}-\delta_{2}-\delta_{3}+\delta_{4},
\end{array}
$$

where $\delta_{1}$ represents a correction common to all modes, $\delta_{2}$ a correction anti-correlated in kaon isospin but blind to lepton flavor, and finally $\delta_{3,4}$ are lepton-universality breaking terms, correlated 
and anti-correlated in kaon isospin, respectively. Our calculation at order $e^{2} p^{2}$ gives $\delta_{1}=0.63 \%$, $\delta_{2}=0.57 \%, \delta_{3}=-0.08 \%, \delta_{4}=-0.12 \%$. On the basis of chiral power counting we expect the higher order corrections to scale with the factor $M_{K}^{2} /\left(4 \pi F_{\pi}\right)^{2} \sim 0.2$ which gives the main contributions to the errors shown in the last column of Table 1.

This error estimate is validated by comparison of the fixed chiral order results with the ones obtained within the "soft-photon factorization" approach of [8], which include a class of higher order chiral corrections (see Table 2). The only anomaly appears to be in the coefficient $\delta_{3}$, where one finds $\delta_{3}:-0.08 \% \rightarrow-0.16 \%$ when going from fixed chiral order to the soft factorization scheme. This can be traced back to the cancellation between the negative contribution from $\mathscr{D}_{3}$ $(-0.31 \%)$ and the positive contribution from $\mathscr{D}_{4-3}(0.23 \%)$. Multiplying these individual pieces by 0.2 gives $\sim 0.06$ and $\sim 0.05$, respectively, which is just the order of magnitude of the shift one sees $(-0.08 \rightarrow-0.16)$.

\begin{tabular}{|l|c|c|c|}
\hline & $\delta_{\mathrm{EM}}^{K \ell}\left(\mathscr{D}_{3}\right)(\%)$ & $\delta_{\mathrm{EM}}^{K \ell}\left(\mathscr{D}_{4-3}\right)(\%)$ & $\delta_{\mathrm{EM}}^{K \ell}(\%)$ \\
\hline$K_{e 3}^{0}$ & 0.41 & 0.59 & 1.0 \\
$K_{e 3}^{ \pm}$ & -0.564 & 0.528 & -0.04 \\
$K_{\mu 3}^{0}$ & 1.57 & 0.04 & 1.61 \\
$K_{\mu 3}^{ \pm}$ & -0.006 & 0.011 & 0.005 \\
\hline
\end{tabular}

Table 2: Electromagnetic corrections to the $K_{\ell 3}$ decay rates taken from [10], using the "soft photon factorization" approach of [8] which includes a part of the higher order terms in the chiral expansion. Comparison with the results shown in the previous table validates the estimate of theoretical uncertainties.

The differential decay distribution can be written in the form

$$
\frac{d \Gamma}{d y d z}=\frac{G_{\mathrm{F}}^{2}\left|V_{u s}\right|^{2} M_{K}^{5} C_{K}^{2}}{128 \pi^{3}} S_{\mathrm{EW}}\left|f_{+}^{K \pi}(0)\right|^{2}\left[\bar{\rho}^{(0)}(y, z)+\delta \bar{\rho}^{\mathrm{EM}}(y, z)\right],
$$

where the Lorentz invariants $y=2 p_{K} \cdot p_{\ell} / M_{K}^{2}=2 E_{\ell} / M_{K}$ and $z=2 p_{\pi} \cdot p_{K} / M_{K}^{2}=2 E_{\pi} / M_{K}$ are related to the energy of the charged lepton and the pion, respectively, measured in the rest frame of the kaon. We emphasize that (2.2) should be the basis to properly determine the momentum dependence of the QCD $K_{\ell 3}$ form factors appearing in $\bar{\rho}^{(0)}(y, z)$. For a further detailed discussion, we refer to [10]. Here we just show the density plots of the ratio $\bar{\rho}^{\mathrm{EM}} / \bar{\rho}^{(0)}$ for $K_{e 3}^{0}, K_{e 3}^{ \pm}, K_{\mu 3}^{0}$ and $K_{\mu 3}^{ \pm}$in the Figures 1 - 4. The theoretical uncertainties discussed above induce an overall uncertainty on $\bar{\rho}^{\mathrm{EM}} / \bar{\rho}^{(0)}$ of about $\pm 0.3 \%$. We wish to stress that the correction to the Dalitz distribution can be locally large $(\sim 10 \%)$ and does not have a definite sign, implying cancellations in the integrated total electromagnetic corrections.

\section{Quark mass ratios and $K_{\ell 3}$ decays}

The theoretical expression for the isospin-breaking parameter of $K_{\ell 3}$ takes the form [8]

$$
\delta_{\mathrm{SU}(2)}^{K^{+} \pi^{0}}=2 \sqrt{3}\left(\varepsilon^{(2)}+\varepsilon_{\mathrm{S}}^{(4)}+\varepsilon_{\mathrm{EM}}^{(4)}+\ldots\right) .
$$

It is dominated by the the lowest-order $\pi^{0}-\eta$ mixing angle $\varepsilon^{(2)}$ which is related to the quark mass ratio $R=\left(m_{s}-\widehat{m}\right) /\left(m_{d}-m_{u}\right)$ by $\varepsilon^{(2)}=\sqrt{3} / 4 R\left(\widehat{m}\right.$ denotes the mean-value of $m_{u}$ and $\left.m_{d}\right)$. 


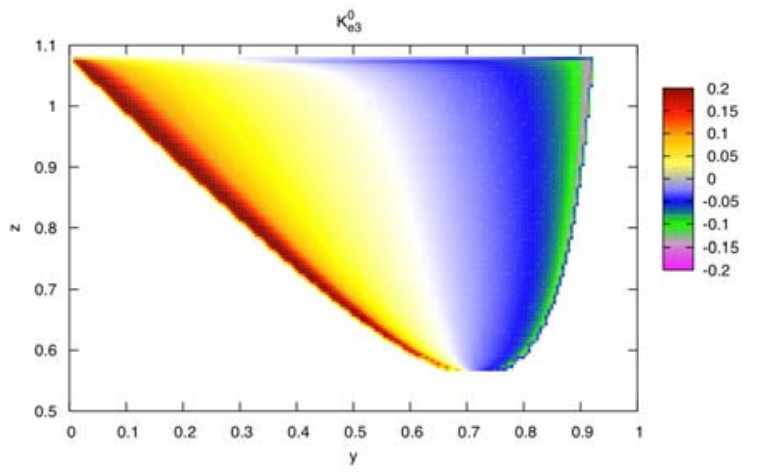

Figure 1: Ratio $\delta \bar{\rho}^{\mathrm{EM}}(y, z) / \bar{\rho}^{(0)}(y, z)$ for $K_{e 3}^{0}$

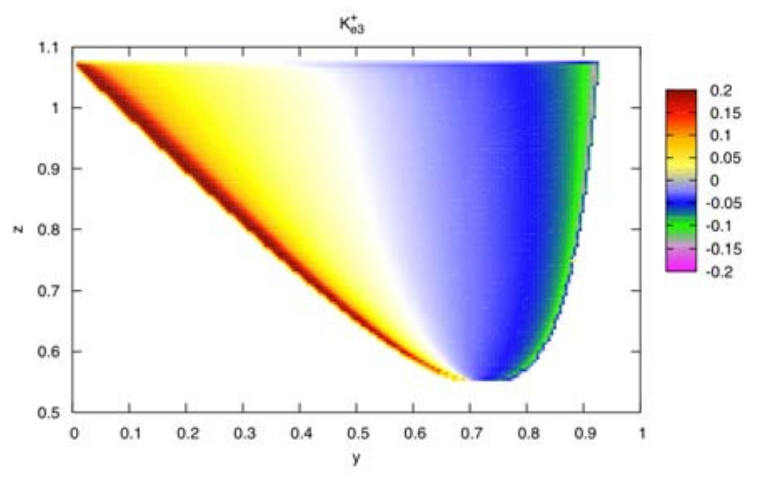

Figure 2: Ratio $\delta \bar{\rho}^{\mathrm{EM}}(y, z) / \bar{\rho}^{(0)}(y, z)$ for $K_{e 3}^{+}$

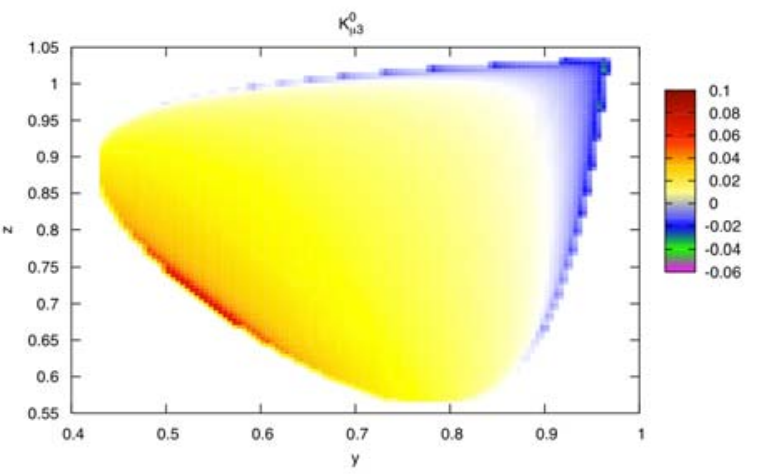

Figure 3: Ratio $\delta \bar{\rho}^{\mathrm{EM}}(y, z) / \bar{\rho}^{(0)}(y, z)$ for $K_{\mu 3}^{0}$ 


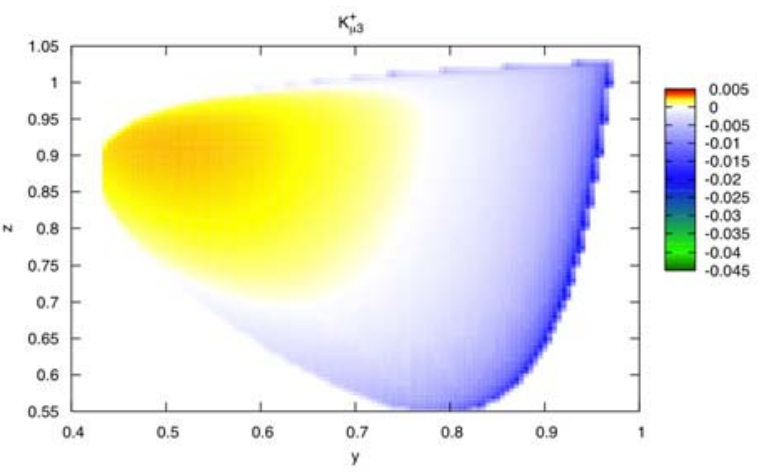

Figure 4: Ratio $\delta \bar{\rho}^{\mathrm{EM}}(y, z) / \bar{\rho}^{(0)}(y, z)$ for $K_{\mu 3}^{+}$

The next-to-leading order corrections $\varepsilon_{\mathrm{S}}^{(4)}$ (order $p^{4}$ ) and $\varepsilon_{\mathrm{EM}}^{(4)}$ (order $e^{2} p^{2}$ ) were computed in [13] and [5], respectively. The explicit expressions for these quantities can be found in [8]. The dots refer to next-to-next-to-leading order contributions (arising at order $p^{6}$ ), the latest results can be found in [14].

The standard strategy up to now has been to use all known information on the ratios of light quarks to predict $\delta_{\mathrm{SU}(2)}$ using Eq. (3.1). However, the present precision of the decay rates and the radiative corrections permits also an "experimental" determination of $\delta_{\mathrm{SU}(2)}$, which can be used as a constraint on the quark mass ratio $R$ via the formula (3.1).

The traditional strategy starts with the observation [15] that the double ratio of quark masses $Q^{2}=\left(m_{s}^{2}-\widehat{m}^{2}\right) /\left(m_{d}^{2}-m_{u}^{2}\right)=R\left(m_{s} / \widehat{m}+1\right) / 2$ can be expressed in terms of meson masses and a purely electromagnetic EM contribution:

$$
Q^{2}=\frac{\Delta_{K \pi} M_{K}^{2}\left(1+\mathscr{O}\left(m_{q}^{2}\right)\right)}{M_{\pi}^{2}\left[\Delta_{K^{0} K^{+}}+\Delta_{\pi^{+} \pi^{0}}-\left(\Delta_{K^{0} K^{+}}+\Delta_{\pi^{+} \pi^{0}}\right)_{\mathrm{EM}}\right]}, \quad \Delta_{P Q}=M_{P}^{2}-M_{Q}^{2} .
$$

The electromagnetic term $\left(\Delta_{K^{0} K^{+}}+\Delta_{\pi^{+} \pi^{0}}\right)_{\mathrm{EM}}$ vanishes to lowest order $e^{2} p^{0}$, a calculation at order $e^{2} p^{2}$ gives $[4,5]$

$$
\begin{aligned}
& \left(\Delta_{K^{0} K^{+}}+\Delta_{\pi^{+} \pi^{0}}\right)_{\mathrm{EM}}=e^{2} M_{K}^{2}\left[\frac{1}{4 \pi^{2}}\left(3 \ln \frac{M_{K}^{2}}{\mu^{2}}-4+2 \ln \frac{M_{K}^{2}}{\mu^{2}}\right)\right. \\
& \left.+\frac{4}{3}\left(K_{5}+K_{6}\right)^{r}(\mu)-8\left(K_{10}+K_{11}\right)^{r}(\mu)+16 Z L_{5}^{r}(\mu)\right]+\mathscr{O}\left(e^{2} M_{\pi}^{2}\right) .
\end{aligned}
$$

The current estimates [11] of the electromagnetic low-energy constants appearing in this expression imply a large deviation from Dashen's limit, $\left(\Delta_{K^{0} K^{+}}+\Delta_{\pi^{+} \pi^{0}}\right)_{\mathrm{EM}}=-1.5 \Delta_{\pi^{+} \pi^{0}}$ [11], which gives $Q=20.7 \pm 1.2$ [16]. Such a small value of $Q$ (compared to $Q=22.7 \pm 0.8$ given by Leutwyler in 1996 [17]) was also supported by other studies $(Q=22.0 \pm 0.6$ in [18] and $Q \simeq 20$ in [19]). It should be noted, however, that the rather large value $Q=23.2$ was obtained from an analysis of $\eta \rightarrow 3 \pi$ at two loops [20]. On the other hand, the determinations of the second input parameter $m_{s} / \widehat{m} \sim 24$ remained rather rather stable over the last years. Combining $Q=20.7 \pm 1.2$ with $m_{s} / \widehat{m}=24.7 \pm 1.1$, we find $R=33.5 \pm 4.3$ and finally $\delta_{\mathrm{SU}(2)}=0.058(8)$ [16]. It is amazing to see that this value is in perfect aggreement with $\delta_{\mathrm{SU}(2) \text { exp. }}=0.054(8)$ obtained from $K_{\ell 3}$ data [1]. 


\begin{tabular}{cccccc}
\hline ISTRA + & $\mathrm{KTeV}$ & $\mathrm{KTeV}$ & $\mathrm{NA} 48$ & $\mathrm{KLOE}$ & $\mathrm{KLOE}$ \\
$K_{\mu 3}^{+}$ & $K_{L \mu 3}$ & $K_{L \mu 3}+K_{L e 3}$ & $K_{L \mu 3}$ & $K_{L \mu 3}$ & $K_{L \mu 3}+K_{L e 3}$ \\
\hline $17.1 \pm 2.2$ & $12.8 \pm 1.8$ & $13.7 \pm 1.3$ & $9.5 \pm 1.4$ & $9.1 \pm 6.5$ & $15.4 \pm 2.2$ \\
\hline
\end{tabular}

Table 3: Experimental results for $\lambda_{0}^{K \pi} \times 10^{3}$

\section{4. $K_{\ell 3}$ scalar form factors}

Recent high-statistics mesurements of the $K_{\ell 3}$ form factor parameters $\lambda_{+}^{\prime}, \lambda_{+}^{\prime \prime}, \lambda_{0}$ are available from ISTRA+ [21], KTeV [22], NA48 [23] and KLOE [24]. Only ISTRA+ has analyzed the charged kaon decay $K^{-} \rightarrow \pi^{0} \mu^{-} v$, whereas the results of the other three experiments are based on $K_{L}$ decays. Table 3 shows the experimental results for the scalar slope parameter $\lambda_{0}^{K \pi}$, defined by

$$
\frac{f_{0}^{K \pi}(t)}{f_{+}^{K \pi}(0)}=1+\lambda_{0}^{K \pi} \frac{t}{M_{\pi^{+}}^{2}}+\ldots
$$

It is difficult to accomodate the result of NA48 with those of the other experiments and the actual value of this quantity is not yet finally settled. A critical review of the these data and a global analysis of the present experimental situation can be found in [1]. Clearly, if the numbers given by ISTRA+ (obtained from charged kaon decays) and NA48 (extracted from $K_{L}$ decays) were both true, this would signal an enormous isospin violation in the scalar form factors of $K_{\ell 3}$.

For a comparison with the experimental outcomes, I present here the results of recent theoretical analyses of isospin breaking effects in the scalar form factors. At lowest nontrivial chiral order $p^{4},\left(m_{d}-m_{u}\right) p^{2}, e^{2} p^{2}$ one obtains the following results for the slope parameters [16]:

$$
\begin{aligned}
\lambda_{0}^{K^{0} \pi^{-}} & =(\underbrace{16.64}_{m_{u}=m_{d}}+\underbrace{0.17}_{m_{u} \neq m_{d}}+\underbrace{0.14}_{\mathrm{EM}}) \times 10^{-3} \\
& =16.95(40)(5) \times 10^{-3} \\
\lambda_{0}^{K^{+} \pi^{0}} & =(\underbrace{16.64}_{m_{u}=m_{d}}-\underbrace{0.12}_{m_{u} \neq m_{d}}-\underbrace{0.08}_{\mathrm{EM}}) \times 10^{-3} \\
& =16.44(39)(4) \times 10^{-3} .
\end{aligned}
$$

The value in the limit of isospin conservation $\left(m_{u}=m_{d}, e=0\right)$ and the contributions generated by strong isospin breaking and electromagnetism are displayed separately. The latter two pieces turn out to be of the same size. In the total results, the first error refers to the uncertainty in the input parameter $F_{K} / F_{\pi} f_{+}(0)$ and the second one to the mixing angle $\varepsilon^{(2)}$. We see that both sources of isospin violation generate only tiny shifts with respect to the result in the in isospin limit, with a splitting of the two slope parameters given by

$$
\Delta \lambda_{0}:=\lambda_{0}^{K^{0} \pi^{-}}-\lambda_{0}^{K^{+} \pi^{0}}=(5.1 \pm 0.9) \times 10^{-4} .
$$

The corrections arising at order $p^{6}$ (in the isospin limit) turn out to be quite sizeable. Combining the two-loop results of chiral perturbation theory [25] with an updated estimate of the necessary 
$p^{6}$ low-energy couplings, the numerical value of the slope parameter in the isospin symmetric limit is given by [16]

$$
\lambda_{0}^{K \pi}=\left(13.9_{-0.4}^{+1.3} \pm 0.4\right) \times 10^{-3} .
$$

The main uncertainty in this result comes from a certain combination of $p^{6}$ low energy couplings which has been determined by an updated analysis based on [26, 27]. Such a reduction of the size of the slope parameter (compared to the next-to-leading order result) is also supported by approaches using dispersive methods. Based on a detailed study of strangeness-changing scalar form factors [28], a recent analysis gives [29] $\lambda_{0}^{K \pi}=(14.7 \pm 0.4) \times 10^{-3}$, being in good agreement with the value (4.5) obtained in chiral perturbation. Using the dispersive representation proposed in [30], we find [16] $\lambda_{0}^{K \pi}=(15.1 \pm 0.8) \times 10^{-3}$, again consistent with (4.5).

The inclusion of isospin violating contributions of order $\left(m_{d}-m_{u}\right) p^{4}$ does not change this picture substantially. We expect an additional uncertainty for the values of the slope parameters of at most $\pm 10^{-3}$, mainly due to not yet fully determined low-energy couplings. Combining the two-loop results given in [14] with an estimate of a further combination of low-energy couplings [16], the difference of the two slope parameters should be confined to the rather small range $0 \lesssim$ $\Delta \lambda_{0} \lesssim 10^{-3}$. In other words, if a difference of the size of the two slope parameters is detected at all, $\lambda_{0}^{K^{0} \pi^{-}}$should be slightly larger than $\lambda_{0}^{K^{+}} \pi^{0}$.

In summary, the experimental results for the scalar slope parameter found by ISTRA+, KTeV and KLOE are in agreement with the predictions of the standard model. On the other hand, the value found by NA48 can hardly be reconciled with our theoretical results. Furthermore, an isospin violation in $\Delta \lambda_{0}$ as it would be suggested by the simultaneous validity of the results of ISTRA+ and NA48 is definitely ruled out within the standard model.

\section{References}

[1] M. Antonelli et al., Flavor physics in the quark sector, arXiv:0907.5386.

[2] A. Sirlin, Current algebra formulation of radiative corrections in gauge theories and the universality of the weak interactions, Rev. Mod. Phys. 50 (1978) 573; Large $m_{W}, m_{Z}$ behaviour of the $O(\alpha)$ corrections to semileptonic processes mediated by W, Nucl. Phys. B 196 (1982) 83.

[3] W. Marciano and A. Sirlin, Radiative corrections to $\pi_{\ell 2}$ decays, Phys. Rev. Lett. 71 (1993) 3629.

[4] R. Urech, Virtual photons in chiral perturbation theory Nucl. Phys. B 433 (1995) 234 [hep-ph/9405341].

[5] H. Neufeld and H. Rupertsberger, Isospin breaking in chiral perturbation theory and the decays $\eta \rightarrow \pi \ell v$ and $\tau \rightarrow \eta \pi v$, Z. Phys. C 68 (1995) 91.

[6] H. Neufeld and H. Rupertsberger, The electromagnetic interaction in chiral perturbation theory, $Z$. Phys. C 71 (1996) 131 [hep-ph/9506448].

[7] M. Knecht, H. Neufeld, H. Rupertsberger and P. Talavera, Chiral perturbation theory with virtual photons and leptons, Eur. Phys. J. C 12 (2000) 469 [hep-ph/9909284].

[8] V. Cirigliano, M. Knecht, H. Neufeld, H. Rupertsberger and P. Talavera, Radiative corrections to $K_{\ell 3}$ decays, Eur. Phys. J. C 23 (2002) 121 [hep-ph/0110153].

[9] V. Cirigliano, H. Neufeld and H. Pichl, $K_{e 3}$ decays and CKM unitarity, Eur. Phys. J. C35 (2004) 53 [hep-ph/0401173]. 
[10] V. Cirigliano, M. Giannotti and H. Neufeld, Electromagnetic effects in $K_{\ell 3}$ decays, JHEP 0811 (2008) 006 [arXiv:0807.4507].

[11] B. Ananthanarayan and B. Moussallam, Four-point correlator constraints on electromagnetic chiral parameters and resonance effective Lagrangians, JHEP 0406 (2004) 047 [hep-ph / 0405206 ].

[12] S. Descotes-Genon and B. Moussallam, Radiative corrections in weak semi-leptonic processes at low energy: A two-step matching determination, Eur. Phys. J. C 42 (2005) 403 [hep-ph/ 0505077 ].

[13] J. Gasser and H. Leutwyler, Low-energy expansion of meson form factors, Nucl. Phys. B 250 (1985) 517.

[14] J. Bijnens and K. Ghorbani, Isospin breaking in $K \pi$ vector form factors for the weak and rare decays $K_{\ell 3}, K \rightarrow \pi \nu \bar{v}$ and $K \rightarrow \pi \ell^{+} \ell^{-}$, arXiv: 0711.0148 .

[15] J. Gasser and H. Leutwyler, Chiral perturbation theory: expansions in the mass of the strange quark, Nucl. Phys. B 250 (1985) 465.

[16] A. Kastner and H. Neufeld, The $K_{\ell 3}$ form factors in the standard model, Eur. Phys. J. C 57 (2008) 541 [arXiv:0805.2222].

[17] H. Leutwyler, The ratios of light quark masses, Phys. Lett B 378 (1996) 313 [hep-ph/9602366].

[18] J. Bijnens and J. Prades, Electromagnetic corrections for pions and kaons: masses and polarizabilities, Nucl. Phys. B 490 (1997) 239 [hep-ph/9610360].

[19] G. Amorós, J. Bijnens and P. Talavera, QCD isospin breaking in meson masses, decay constants and quark mass ratios, Nucl. Phys. B 602 (2001) 87 [hep-ph/0101127].

[20] J. Bijnens and K. Ghorbani, $\eta \rightarrow 3 \pi$ at two loops in chiral perturbation theory, JHEP 0711 (2007) 030 [arXiv:0709.0230].

[21] O.P. Yushchenko et al., High statistics study of the $K^{-} \rightarrow \pi^{0} \mu^{-} v$ decay, Phys. Lett. B 581 (2004) 31 [hep-ex/0312004].

[22] T. Alexopoulos et al., Measurement of semileptonic $K_{L}$ decay form factors, Phys. Rev. D 70 (2004) 092007 [hep-ex/0406003].

[23] A. Lai et al., Measurement of $K_{\mu 3}^{0}$ form factors, Phys. Lett. B 647 (2007) 341 [hep-ex/ 0703002 ].

[24] F. Ambrosino et al., Measurement of the $K_{L} \rightarrow \pi \mu \nu$ form factor parameters with the KLOE detector, JHEP 0712 (2007) 105 [arXiv : 0710 . 4470].

[25] J. Bijnens, P. Talavera, $K_{\ell 3}$ decays in chiral perturbation theory, Nucl. Phys. B 669 (2003) 341 [hep-ph/0303103].

[26] V. Cirigliano et al., The $\langle S P P\rangle$ Green function and $S U(3)$ breaking in $K_{\ell 3}$ decays, JHEP 0504 (2005) 006 [hep-ph/0503108].

[27] V. Cirigliano, A. Pich, G. Ecker, H. Neufeld, Meson resonances, large $N_{c}$ and chiral symmetry, JHEP 0306, (2003) 012 [hep-ph/ 0305311$].$

[28] M. Jamin, J. Oller, A. Pich, Strangeness changing scalar form factors, Nucl. Phys. B 622 (2002) 279 [hep-ph/0110193].

[29] M. Jamin, J.A. Oller, A. Pich, Scalar K $\pi$ form factor and light quark masses, Phys. Rev. D 74 (2006) 074009 [hep-ph/ 0605095$].$

[30] V. Bernard, M. Oertel, E. Passemar, J. Stern, $K_{\mu 3}^{L}$ decay: A stringent test of right-handed quark currents, Phys. Lett. B B 638 (2006) 480 [hep-ph / 0603202$].$ 